J. Korean Math. Soc. 49 (2012), No. 3, pp. 605-622

http://dx.doi.org/10.4134/JKMS.2012.49.3.605

\title{
RINGS CLOSE TO SEMIREGULAR
}

\author{
Pinar AydoĞdu, Yang Lee, And A. Çı̆̆dem Özcan
}

\begin{abstract}
A ring $R$ is called semiregular if $R / J$ is regular and idempotents lift modulo $J$, where $J$ denotes the Jacobson radical of $R$. We give some characterizations of rings $R$ such that idempotents lift modulo $J$, and $R / J$ satisfies one of the following conditions: (one-sided) unitregular, strongly regular, (unit, strongly, weakly) $\pi$-regular.
\end{abstract}

\section{Introduction}

Throughout this paper, $R$ denotes an associative ring with identity, and all modules are unitary right $R$-modules.

Recall that an element $a$ of a ring $R$ is called regular if there exists $b \in R$ such that $a=a b a . \quad R$ is said to be (von Neumann) regular if every element of $R$ is regular. $R$ is called semiregular if $R / J$ is regular and idempotents lift modulo $J$ (i.e., if, whenever $a^{2}-a \in J$, there exists $e^{2}=e \in R$ such that $e-a \in J)$, where $J=J(R)$ denotes the Jacobson radical of $R$. The well-known characterization of a semiregular ring can be given as follows:

Theorem 1.1 ([20], [21, Theorem 28]). The following are equivalent for a ring $R$ :

(1) $R$ is semiregular.

(2) For any $a \in R$, there exists a regular element $d$ in $R$ such that $a-d \in J$.

(3) For any $a \in R$, there exists a regular element $d \in a R$ (resp. $d \in a R a)$ such that $a-d \in J$.

(4) For any $a \in R$, there exists an idempotent $e \in a R$ such that $(1-e) a \in J$.

(5) For any $a \in R$, there exists an idempotent $e \in R a$ such that $a(1-e) \in J$.

(6) For any $a \in R, R / a R$ has a projective cover.

(7) For any $a \in R, R / R a$ has a projective cover. When these conditions hold,

(8) eRe is semiregular for every $e^{2}=e \in R$.

(9) $R / I$ is semiregular for every ideal $I$ of $R$.

Received November 9, 2010.

2010 Mathematics Subject Classification. 16E50, 16 U99.

Key words and phrases. idempotent lifting, semi unit-regular ring, semi (strongly) $\pi$ regular ring.

(C)2012 The Korean Mathematical Society 
In the following proper implications, we see the rings close to regular. Their definitions can be found in the text.

strongly regular $\Rightarrow$ unit-regular $\Rightarrow$ one-sided unit-regular $\Rightarrow$ regular $\Rightarrow$ weakly regular,

strongly regular $\Rightarrow$ strongly $\pi$-regular $\Rightarrow$ unit $\pi$-regular $\Rightarrow \pi$-regular $\Rightarrow$ weakly $\pi$-regular.

In this article, we investigate rings close to semiregular by the motivation of the characterization of semiregular rings and the rings mentioned above.

We call a ring $R$ semi (*)-regular if idempotents lift modulo $J$, and $R / J$ satisfies $(*)$, where $(*)$ is (one-sided) unit-regular or strongly regular or (unit, strongly, weakly) $\pi$-regular.

In Section 2, we give several characterizations of semi (one-sided) unitregular and semi strongly regular rings. We prove among other things that $R$ is semi one-sided unit-regular if and only if there exists a complete orthogonal set $\left\{e_{1}, \ldots, e_{n}\right\}$ of idempotents of $R$ such that all $e_{i} R e_{j}$ are semi one-sided unit-regular.

Section 3 is concerned with $\pi$-regularity. We give some characterizations of semi strongly $\pi$-regular and semi unit $\pi$-regular rings as in Section 2 . Also, we consider a generalization of weak $\pi$-regularity and investigate the relationship between this generalization and semi weak $\pi$-regularity. In addition, we obtain a characterization of a ring $R$ such that $R / J$ is Eulerian and idempotents lift modulo $J$, among others. Furthermore, we show that some rings, we are interested in, coincide if the ring is abelian or right quasi-duo. Although semi $\pi$-regular rings are exchange rings, semi weakly $\pi$-regular rings need not be exchange rings. We give an example in order to support this fact.

Following Crawley and Jonsson [11], a module $M$ is said to have the ( $f u l l)$ exchange property if for any module $X$ and decompositions $X=M^{\prime} \oplus Y=$ $\oplus_{i \in I} N_{i}$, where $M^{\prime} \cong M$, there exist submodules $N_{i}^{\prime} \subseteq N_{i}$ for each $i$ such that $X=M^{\prime} \oplus\left(\oplus N_{i}^{\prime}\right)$. If this condition holds for finite sets $I$ (equivalently for $|I|=2)$, the module $M$ is said to have the finite exchange property. Warfield [25] calls a ring $R$ exchange if $R$ has the exchange property as a right $R$ module. Also, it is proved in [25] that the notion of exchange rings is left-right symmetric.

\section{Semi (one-sided) unit-regular, semi strongly regular rings}

In this section, we give some characterizations of semi (one-sided) unitregular and semi strongly regular rings. We begin with unit-regularity. An element $a$ of $R$ is called unit-regular if there exists a unit $u \in R$ such that $a=a u a$. $R$ is called unit-regular if every element of $R$ is unit-regular.

Recall that a ring $R$ is said to have stable range 1 if, for any $a, b \in R$ satisfying $a R+b R=R$, there exists $y \in R$ such that $a+b y$ is a (right) unit [24]. We know from [12] that unit-regular rings have stable range 1. Also, $R$ has stable range 1 if and only if $R / J$ has stable range 1 by [24]. If $R$ has 
stable range 1 , then every regular element is unit-regular by [12, the proof of Proposition 4.12] or [4]. The converse is true if $R$ is an exchange ring [4, Theorem 3].

Chen [8] calls a ring $R$ strongly stable if, whenever $a R+b R=R$, there exists $w \in Q(R)$ such that $a+b w$ is unit in $R$, where $Q(R)=\left\{x \in R: \exists e-e^{2} \in J\right.$ and a unit $u$ such that $x=e u\}$.

The following theorem states several characterizations of semiregular rings with stable range one or semiregular strongly stable rings.

Theorem 2.1. The following are equivalent for a ring $R$ :

(1) $R / J$ is unit-regular and idempotents lift modulo $J$.

(2) For any $a \in R$, there exists a unit-regular element $d$ in $R$ such that $a-d \in J$.

(3) For any $a \in R$, there exists a unit-regular element $d \in a R$ (resp. $d \in$ $a R a)$ such that $a-d \in J$.

(4) For any $a \in R$, there exist an idempotent $e$ and $a$ unit $b$ in $R$ such that $e \in a R,(1-e) a \in J$ and $b a-(b a)^{2} \in J$.

(5) For any $a \in R$, there exist an idempotent $e$ and $a$ unit $b$ in $R$ such that $e \in R a, a(1-e) \in J$ and $a b-(a b)^{2} \in J$.

(6) For any $a \in R, R / a R$ has a projective cover and $\bar{R} / \bar{a} \bar{R} \cong r_{\bar{R}}(\bar{a})$ as right $\bar{R}$-modules, where $\bar{R}=R / J$ and $r_{\bar{R}}$ is the right annihilator.

(7) For any $a \in R, R / R a$ has a projective cover and $\bar{R} / \bar{R} \bar{a} \cong l_{\bar{R}}(\bar{a})$ as left $\bar{R}$-modules, where $\bar{R}=R / J$ and $l_{\bar{R}}$ is the left annihilator.

(8) $R$ is a semiregular ring with stable range 1 .

(9) $R$ is a semiregular strongly stable ring.

Proof. $(1) \Rightarrow(2)$ Since $R / J$ is unit-regular, it has stable range 1 . This implies that $R$ has stable range 1 and hence every regular element of $R$ is unit-regular. By Theorem 1.1, (2) holds.

$(2) \Rightarrow(1)$ It is obvious.

(1) $\Leftrightarrow$ (3) Similar to that of $(1) \Leftrightarrow(2)$.

$(1) \Rightarrow(4)$ Let $a \in R$. Then there exists a unit $b$ in $R$ such that $a-a b a \in J$. So $b a-(b a)^{2} \in J$. By hypothesis, there exists an idempotent $f \in R$ such that $f-b a \in J$. Then $1-f+b a=u$ is a unit in $R$. If we let $e=a u^{-1} f b$, then we obtain that $e^{2}=e \in a R b$. Since $u+J=\bar{u}=\overline{1}$, we have that $\bar{a} \bar{f}=\bar{a} \bar{b} \bar{a}=\bar{a}$. This implies that $\bar{a}-\overline{e a}=\overline{0}$. Hence, $(1-e) a \in J$.

$(4) \Rightarrow(1)$ Let $a \in R$. Choose $e$ and $b$ as in (4). Since $e \in a R=a R b, R$ is semiregular by Theorem 1.1. Let $e=a r b$, where $r \in R$. Since $(1-e) a \in J$, $\bar{a}=\overline{e a}=\overline{a r} \bar{b} \bar{a}$. Multiplying this equation by $\bar{b} \bar{a}$ from the right, we have $\bar{a} \bar{b} \bar{a}=\bar{a}$, where $\bar{b}$ is a unit. Hence, $R / J$ is unit-regular.

(1) $\Leftrightarrow(5)$ Follows from the symmetry of the condition (1).

$(1) \Leftrightarrow(6)$ It is well-known that $R$ is unit-regular if and only if $a R$ is a direct summand of $R$ and $R / a R \cong r_{R}(a)$ as right $R$-modules for every $a \in R$.

$(1) \Leftrightarrow(7)$ Follows from the symmetry of the condition (1).

$(1) \Rightarrow(8)$ Since $R / J$ has stable range $1, R$ has stable range 1 . 
$(8) \Rightarrow(1)$ Since $R / J$ has stable range 1 and is regular, it is unit-regular by [12, Proposition 4.12].

$(8) \Leftrightarrow(9)$ By $[8$, p. 2774], it is obvious.

We call an element $a \in R$ semi unit-regular if $a$ satisfies the condition (2) of Theorem 2.1. A ring $R$ is called semi unit-regular if $R$ satisfies one of the equivalent conditions of Theorem 2.1 (see also [5]). Also, Chen calls this class of rings unit semiregular in [8]. In the following we see a semi unit-regular ring but not (unit-)regular.

Example 2.2. Let $K$ be a field and $R=K[[x]]$ be the (formal) power series ring with indeterminate $x$ over $K$. Note that $R$ is not $\pi$-regular and $J=$ $x K[[x]]$. So $R / J \cong K$ is unit-regular. Let $f(x)^{2}-f(x) \in J$ and $f(x)=$ $a_{0}+a_{1} x+\cdots \in R$. Then $a_{0}^{2}=a_{0}$ and this yields that $a_{0}=0$ or $a_{0}=1$. When $a_{0}=0,0-f(x) \in J$. When $a_{0}=1, f(x)=1+a_{1} x+\cdots$ and so $1-f(x)=a_{1} x+\cdots \in J$. These imply that idempotents lift modulo $J$.

The following are easy consequences of Theorem 2.1.

Corollary 2.3. If $a-b \in J$ and $b$ is semi unit-regular, then $a$ is semi unitregular.

Corollary 2.4. If $R$ is semi unit-regular, then so is every homomorphic image of $R$ and so is every subring of the form eRe, where $e^{2}=e \in R$.

Proof. Let $I$ be an ideal of $R$ and $\bar{a} \in R / I$. By Theorem 2.1, there exists a unitregular element $d \in R$ such that $a-d \in J$. Then $\bar{a}-\bar{d} \in(J+I) / I \subseteq J(R / I)$ and $\bar{d}$ is unit-regular in $R / I$. Hence, $R / I$ is semi unit-regular.

Let $e$ be an idempotent of $R$. Then $e R e$ is semiregular by [20, Lemma B.42] and $e R e$ has stable range 1 by [8, Lemma 3.2]. It follows that $e R e$ is semi unit-regular.

$\mathrm{Wu}[26]$ defines rings with weak stable range 1 by considering one-sided units instead of units in the definition of rings with stable range 1 . That is, a ring $R$ is said to have weak stable range 1 if, for any $a, b, x \in R$ satisfying $a x+b=1$, there exists an element $y$ in $R$ such that $a+b y$ is a one-sided unit.

If $R$ is an exchange ring, then $R$ has weak stable range 1 if and only if every regular element of $R$ is one-sided unit-regular by [18] and [27].

A ring $R$ is called directly finite if all one-sided inverses are two-sided, i.e., if $a b=1$ for any $a, b \in R$, then $b a=1$. Hence, $R$ has stable range 1 if and only if $R$ has weak stable range 1 and is directly finite.

As we mentioned before, it is known that a ring $R$ has stable range 1 if and only if $R / J$ has stable range 1 . A similar result holds for rings which have weak stable range 1:

Lemma 2.5. Let $I$ be an ideal of a ring $R$ such that $I \subseteq J$. $R$ has weak stable range 1 if and only if $R / I$ has weak stable range 1 . 
Proof. $(\Rightarrow)$ Let $\bar{a}, \bar{b}, \bar{x} \in \bar{R}=R / I$ satisfying $\overline{a x}+\bar{b}=\overline{1}$. Since $I \subseteq J$, $a x+b$ is a unit in $R$. Let $u$ be in $R$ such that $(a x+b) u=1$. By hypothesis, there exists $y \in R$ such that $a+b u y$ is a one-sided unit. Hence, $\bar{a}+\bar{b} \overline{u y}$ is a one-sided unit.

$(\Leftarrow)$ Let $a, b, x \in R$ such that $a x+b=1$. Since $\bar{R}$ has weak stable range 1 , there exists $\bar{y} \in \bar{R}$ such that $\bar{a}+\bar{b} \bar{y}$ is a one-sided unit. Assume that $\bar{a}+\bar{b} \bar{y}$ is a right unit. Then there exists $u \in R$ such that $1-(a+b y) u \in I \subseteq J$. This implies that $a+b y$ is a right unit.

Corollary 2.6. $R$ has weak stable range 1 if and only if $R / J$ has weak stable range 1.

Using the idea of Vaserstein's proof given in [24, Theorem 2.8] we obtain the following lemma.

Lemma 2.7. If $R$ has weak stable range 1, then eRe has weak stable range 1 for any idempotent e of $R$.

Proof. Let $a, b, x \in S=e$ Re such that $a x+b=e$. Then $a S+b S=S$. Consider $a+1-e$ and $b$ in $R$. Since $(1-e) S=0$, we have $e \in a S+b S \subseteq(a+1-e) R+b R$. But $a(1-e)=0=b(1-e)$ implies that $1-e=(a+1-e)(1-e)+b(1-e) \in$ $(a+1-e) R+b R$. Hence, we have $(a+1-e) R+b R=R$. Let $y, z \in R$ such that $(a+1-e) y+b z=1$. There exists $t \in R$ such that $(a+1-e)+b z t$ is a one-sided unit. Assume that it is a right unit. Since $[1-b z t(1-e)][1+b z t(1-e)]=1$, we have that $R=(1-b z t(1-e))(a+(1-e)+b z t) R=(a+(1-e)+b z t e) R$. This implies that $S=(a+b z t e) S$. Hence, there exists an element $y \in S$ such that $a+b y$ is a right unit. Similarly, if $(a+1-e)+b z t$ is a left unit in $R$, then there exists an element $y \in S$ such that $a+b y$ is a left unit.

The next theorem characterizes semiregular rings with weak stable range 1 .

Theorem 2.8. The following are equivalent for a ring $R$ :

(1) $R / J$ is one-sided unit-regular and idempotents lift modulo $J$.

(2) For any $a \in R$, there exists a one-sided unit-regular element $d \in R$ such that $a-d \in J$.

(3) For any $a \in R$, there exists a one-sided unit-regular element $d \in a R$ (resp. $d \in a R a$ ) such that $a-d \in J$.

(4) $R$ is a semiregular ring with weak stable range 1.

(5) For any $a \in R$, there exist a one-sided unit $b \in R$ and an idempotent $e \in a R b$ such that $(1-e) a \in J$ and $b a-(b a)^{2} \in J$.

(6) For any $a \in R$, there exist $a$ one-sided unit $b \in R$ and an idempotent $e \in b R a$ such that $a(1-e) \in J$ and $a b-(a b)^{2} \in J$.

Proof. (2) $\Rightarrow$ (1) Since $R$ is semiregular, idempotents lift modulo $J$.

$(1) \Rightarrow(2)$ Since $R$ is semiregular, $R / J$ is an exchange ring by $[25$, Theorem 3]. Hence, by [18] and Corollary $2.6, R$ has weak stable range 1 . Again by [18], every regular element of $R$ is one-sided unit-regular in $R$. Hence, by Theorem 1.1, (2) holds. 
(1) $\Leftrightarrow$ (3) Similar to that of $(1) \Leftrightarrow(2)$.

$(1) \Rightarrow(4)$ By the proof of $(1) \Rightarrow(2), R$ has weak stable range 1 .

$(4) \Rightarrow(1)$ By [18] and [27], every regular element is a one-sided regular element.

$(1) \Leftrightarrow(5)$ and $(1) \Leftrightarrow(6)$ can be seen by a proof similar to that of $(1) \Leftrightarrow(4)$ in Theorem 2.1.

An element $a \in R$ is called semi one-sided unit-regular if it satisfies the condition (2) in Theorem 2.8. A ring $R$ is called a semi one-sided unit-regular ring if $R$ satisfies one of the equivalent conditions of Theorem 2.8. Example 2.2 provides a semi one-sided unit-regular ring but not one-sided unit-regular.

Corollary 2.9. If $a-b \in J$ and $b$ is semi one-sided unit-regular, then $a$ is semi one-sided unit-regular.

Corollary 2.10. If $R$ is a semi one-sided unit-regular ring, then so is every homomorphic image of $R$ and so is every subring of the form $e$ Re, where $e^{2}=e$.

Proof. Follows from Theorem 2.8, Lemma 2.7 and the proof of Corollary 2.4.

According to [9, Theorem 3.5], $R$ is semi unit-regular if and only if there exists a complete orthogonal set $\left\{e_{1}, \ldots, e_{n}\right\}$ of idempotents of $R$ such that all $e_{i} R e_{j}$ are semi unit-regular. We show that a similar result is also valid for semi one-sided unit-regular rings. Before proving this result we need the following lemma.

Following [27], a module $M$ is said to satisfy outer weak cancellation if $M \oplus$ $K \cong M \oplus L$ implies that there exists a splitting epimorphism between $K$ and $L$.

Lemma 2.11. Let $M=M_{1} \oplus M_{2}$. If $M_{1}$ and $M_{2}$ satisfy outer weak cancellation, then so does $M$.

Proof. Let $M_{1} \oplus M_{2} \oplus K \cong M_{1} \oplus M_{2} \oplus L$. Since $M_{1}$ satisfies outer weak cancellation, there exists a splitting epimorphism $f: M_{2} \oplus K \rightarrow M_{2} \oplus L$. Let $g: M_{2} \oplus L \rightarrow M_{2} \oplus K$ be the monomorphism such that $f g=1_{M_{2} \oplus L}$. Then we obtain that $M_{2} \oplus K=\operatorname{Ker} f \oplus \operatorname{Im} g$. It follows that $M_{2} \oplus K \cong M_{2} \oplus(\operatorname{Ker} f \oplus L)$ and hence there exists a splitting epimorphism $\alpha: K \rightarrow \operatorname{Ker} f \oplus L$, because $M_{2}$ satisfies outer weak cancellation. Thus, $\pi \alpha: K \rightarrow L$ is a splitting epimorphism, where $\pi$ is the projection from $\operatorname{Ker} f \oplus L$ onto $L$.

By induction we obtain the following result.

Corollary 2.12. Let $M=\oplus_{i=1}^{n} M_{i}$. If $M_{i}$ satisfies outer weak cancellation for all $i=1, \ldots, n$, then so does $M$.

In [18], it is proved that if $M$ has the finite exchange property, then $M$ satisfies outer weak cancellation if and only if $\operatorname{End}_{R}(M)$ has weak stable range 1. Considering this fact, we now prove the result mentioned above. 
Theorem 2.13. The following are equivalent for a ring $R$ :

(1) $R$ is semi one-sided unit-regular.

(2) There exists a complete orthogonal set $\left\{e_{1}, \ldots, e_{n}\right\}$ of idempotents of $R$ such that all $e_{i} R e_{j}$ are semi one-sided unit-regular.

Proof. (1) $\Rightarrow(2)$ Trivial by taking $e=1$.

$(2) \Rightarrow(1)$ By $\left[9\right.$, Theorem 2.2], $R$ is semiregular. By hypothesis, $\operatorname{End}\left(e_{i} R\right) \cong$ $e_{i} R e_{i}$ is semiregular and has weak stable range 1 for all $i=1, \ldots, n$. It follows from [25, Theorem 2] that all $e_{i} R$ have the finite exchange property and so all $e_{i} R$ satisfy outer weak cancellation. By Corollary 2.12, $\oplus_{i=1}^{n} e_{i} R$ satisfies outer weak cancellation and hence $\operatorname{End}\left(\oplus_{i=1}^{n} e_{i} R\right)$ has weak stable range 1 , because $R \cong e_{1} R \oplus \ldots \oplus e_{n} R$ has the finite exchange property. Thus, $R$ is semi one-sided unit-regular.

In the final part of this section, we consider strongly regular rings.

A ring $R$ is called strongly regular if, for any $a \in R$, there exists $x \in R$ such that $a=a^{2} x$ (see [2]). A ring $R$ is called abelian if all idempotents of $R$ are central. It is well-known that $R$ is strongly regular if and only if $R$ is regular and abelian, if and only if $R$ is unit-regular and abelian, if and only if, for any $a \in R$, there exist an idempotent $e$ and a unit $u$ in $R$ such that $a=e u$ and $e u=u e$.

According to Nicholson and Zhou [21], idempotents lift strongly modulo $J$ if, whenever $a^{2}-a \in J$, there exists $e^{2}=e \in a R(R a, a R a)$ such that $e-a \in J$. They prove that if idempotents lift modulo $J$, then they lift strongly modulo $J$ [21, Lemma 5]. Now we have the following result.

Theorem 2.14. The following are equivalent for a ring $R$ :

(1) $R / J$ is strongly regular and idempotents lift modulo $J$.

(2) For any $a \in R$, there exist $a$ unit $u$ and an idempotent e in $R$ (resp. in $a R)$ such that $a-e u \in J$ and $e u-u e \in J$.

(3) For any $a \in R$, there exist $a$ unit $u$ and an idempotent $e \in a R$ such that $(1-e) a \in J$ and $\overline{u a}=\overline{a u}$ is an idempotent of $R / J$.

(4) For any $a \in R$, there exist $a$ unit $u$ and an idempotent $e \in R a$ such that $a(1-e) \in J$ and $\overline{a u}=\overline{u a}$ is an idempotent of $R / J$.

Proof. (1) $\Rightarrow(2)$ By Theorem 2.1, for any $a \in R$, there exists a unit-regular element $d$ in $R$ ( $a R$ or $a R a$ ) such that $a-d \in J$. Then $d=e u$, where $e$ is an idempotent and $u$ is a unit. Since $R / J$ is abelian, $e u-u e \in J$.

$(2) \Rightarrow(1)$ If $u$ is a unit and $e$ is an idempotent, then $e u$ is unit-regular. Hence, by Theorem 2.1, idempotents lift modulo $J$. By the characterization of a strongly regular ring written above, $R / J$ is strongly regular.

$(1) \Rightarrow(3)$ Let $\bar{a} \in R / J$. Since $R / J$ is strongly regular, there exist an idempotent $\bar{e}$ and a unit $\bar{u}$ in $R / J$ such that $\bar{a}=\overline{e u}$ and $\overline{e u}=\overline{u e}$. It follows that $\bar{a}=\overline{e a}$ and $\overline{a u}=\overline{u a}$ is an idempotent of $R / J$. Since $\bar{e}=\overline{a u}^{-1}$ and idempotents lift strongly modulo $J, e$ can be assumed to be an idempotent in $a R$. 
$(3) \Rightarrow(1)$ By Theorem 2.1, idempotents lift modulo $J$. Let $\bar{a} \in R / J$. Choose $e$ and $u$ as in (3). Then there exists $r \in R$ such that $e=a r u$. We obtain that $\bar{a}=\overline{e a}=\overline{a r u a}$ and so $\overline{a u a}=\overline{a r u a}=\bar{a}=\bar{a}^{2} \bar{u}$. Hence, $R / J$ is strongly regular.

$(1) \Leftrightarrow(4)$ Follows from the symmetry of the condition (1).

We call a ring $R$ semi strongly regular if $R$ satisfies one of the equivalent conditions of Theorem 2.14. One can easily obtain that if, for any $a \in R$, there exists a strongly regular element $d$ (i.e., $d=d^{2} x$ for some $x \in R$ and $d x=x d$ ) such that $a-d \in J$, then $R$ is semi strongly regular. Example 2.2 provides a semi strongly regular ring but not regular.

Corollary 2.15. If $R$ is a semi strongly regular ring, then so is every homomorphic image of $R$ and so is every subring of the form eRe, where $e^{2}=e$.

Proof. Let $I$ be an ideal of $R$. Since $R$ is semiregular, $\bar{R}=R / I$ is semiregular. Therefore idempotents lift modulo $J(\bar{R})$. Note that any homomorphic image of a strongly regular ring is strongly regular. Since $R / J$ is strongly regular and $\bar{R} / J(\bar{R})$ is a homomorphic image of $R / J$, we obtain that $\bar{R}$ is semi strongly regular.

Let $e$ be an idempotent of $R$. Since $R$ is semiregular, idempotents lift modulo $J(e R e)$. By the definition of strongly regular rings, we observe that if a ring $R$ is strongly regular, then $e R e$ is strongly regular for any idempotent $e \in R$. Hence, $R / J$ being strongly regular implies that $\bar{e}(R / J) \bar{e} \cong e R e / J(e R e)$ is strongly regular.

A ring $R$ is said to have unit stable range 1 if, for any $a, b \in R$ satisfying $a R+b R=R$, there exists a unit $u \in R$ such that $a+b u$ is a unit. In contrast to Theorems 2.1 and 2.8, there exists a semi strongly regular ring which does not have unit stable range one. The ring $\mathbb{Z}_{2}$ is semi strongly regular with stable range 1 , but does not have unit stable range 1 . On the other hand, $\mathrm{M}_{2}\left(\mathbb{Z}_{2}\right)$, the ring of $2 \times 2$ matrices over $\mathbb{Z}_{2}$, is semiregular and by [7, Corollary 4], has unit stable range 1 . But it is not semi strongly regular since it has non-central idempotents.

A ring $R$ is called right weakly regular if $B^{2}=B$ for every principal right ideal $B$ of $R$ (see $[23,4.4]$ ). It is obvious that semi strongly regular $\Rightarrow$ semi unit-regular $\Rightarrow$ semi one-sided unit-regular $\Rightarrow$ semiregular $\Rightarrow$ semi right weakly regular (i.e., $R / J$ is right weakly regular and idempotents lift modulo $J$ ). But none of the implications are reversible because, for example, it is known that there exists a unit-regular ring which is not strongly regular. For the last implication, there exists a right weakly regular ring with Jacobson radical zero which is not regular (see [22]).

\section{Semi (unit, strongly, weakly) $\pi$-regular rings}

This section is concerned with $\pi$-regularity. We characterize semi $\pi$-regular, semi unit $\pi$-regular, semi strongly $\pi$-regular rings, respectively. Rings $R$ such that $R / J$ is eulerian and idempotents lift modulo $J$ are also considered. 
An element $a$ of a ring $R$ is called $\pi$-regular if a power of $a$ is regular. A ring $R$ is called $\pi$-regular if every element of $R$ is $\pi$-regular. Due to [28], an element $a$ of a ring $R$ is called semi $\pi$-regular if there exists a positive integer $n$ such that $a^{n}$ is semiregular, i.e., $a^{n}$ satisfies the condition (4) in Theorem 1.1 (see [20, Lemma B.40]). A ring $R$ is called semi $\pi$-regular if every element of $R$ is semi $\pi$-regular. Also, by [28, Proposition 4.1] and [28, Theorem 4.4], $R$ is semi $\pi$-regular if and only if, for any $a \in R$, there exist a positive integer $n$ and a regular element $b \in R$ such that $a^{n}-b \in J$, if and only if $R / J$ is $\pi$-regular and idempotents can be lifted modulo $J$. We will use these equivalences freely. It is clear that $\pi$-regular rings are semi $\pi$-regular, but the converse need not hold by Example 2.2. We have the following characterization.

Theorem 3.1. The following are equivalent for a ring $R$ :

(1) $R$ is semi $\pi$-regular.

(2) For any $a \in R$, there exist a positive integer $n$ and a $\pi$-regular element $d$ of $R$ such that $a^{n}-d \in J$.

(3) For any $a \in R$, there exist a positive integer $n$ and a $\pi$-regular element $d \in a^{n} R$ (resp. $\left.d \in a^{n} R a^{n}\right)$ such that $a^{n}-d \in J$.

Proof. Write $\bar{x}=x+J$. (1) $\Rightarrow(3)$ Let $a \in R$. By [28, Proposition 4.1], there exist a positive integer $n$ and $b \in R$ such that $b=b a^{n} b$ and $a^{n}-a^{n} b a^{n} \in J$. Let $d=a^{n} b a^{n}$. Then $d=d b d$ and $a^{n}-d \in J$.

$(3) \Rightarrow(2)$ It is obvious.

$(2) \Rightarrow(1)$ Let $a \in R, n$ be a positive integer and $d$ be a $\pi$-regular element of $R$ such that $a^{n}-d \in J$. Then there exists a positive integer $m$ such that $d^{m}$ is regular. Since $\bar{a}^{n m}=\bar{d}^{m}, a^{n m}-d^{m} \in J$. By [28, Theorem 4.4], (1) holds.

The $n$ by $n$ upper triangular matrix ring over a $\operatorname{ring} R$ is denoted by $U_{n}(R)$. Define $D_{n}(R)=\left\{a \in U_{n}(R) \mid\right.$ all diagonal entries of $a$ are equal $\}$ and $V_{n}(R)=$ $\left\{b=\left(b_{i j}\right) \in D_{n}(R) \mid b_{s t}=b_{(s+1)(t+1)}\right.$ for $s=1, \ldots, n-2$ and $\left.t=2, \ldots, n-1\right\}$.

Corollary 3.2. (1) A ring $R$ is semi $\pi$-regular if and only if $D_{n}(R)$ is semi $\pi$-regular if and only if $V_{n}(R)$ is semi $\pi$-regular.

(2) A ring $R$ is semi $\pi$-regular if and only if so is $R[x] /\left\langle x^{n}\right\rangle$, where $R[x]$ is the polynomial ring with an indeterminate $x$ over $R$ and $\left\langle x^{n}\right\rangle$ is the ideal of $R[x]$ generated by $x^{n}$.

Proof. (1) The proof is obtained from Theorem 3.1 and the fact that $J\left(D_{n}(R)\right)$ $=\left\{c=\left(c_{i j}\right) \in D_{n}(R) \mid c_{i j} \in J(R)\right\}$ and $J\left(V_{n}(R)\right)=\left\{d=\left(d_{i j}\right) \in D_{n}(R) \mid d_{i j} \in\right.$ $J(R)\}$. (2) The proof is obtained from (1) and $V_{n}(R) \cong R[x] /\left\langle x^{n}\right\rangle$.

We know from [28] that if $R$ is a semi $\pi$-regular ring, then so is every homomorphic image of $R$ and so is every subring of the form $e R e$, where $e^{2}=e$. It is obvious that semiregular rings are semi $\pi$-regular, but the converse need not hold by the following. The $n$ by $n$ full matrix ring over a $\operatorname{ring} R$ is denoted by $\operatorname{Mat}_{n}(R)$. 
Example 3.3. Let $K$ be a field and consider a ring

$R=\left\{\left(a_{i}\right)_{i=1}^{\infty} \mid a_{i} \in \operatorname{Mat}_{n}(K)\right.$ for all $i$ and $a_{i}$ is eventually in $\left.U_{n}(K)\right\}$,

where $\left(a_{i}\right)_{i=1}^{\infty}$ is a sequence. Then $R$ is $\pi$-regular but not regular, through well-known facts. Note $J=0$. These imply that $R$ is semi $\pi$-regular but not semi-regular.

An element $a \in R$ is called unit $\pi$-regular if there exists a positive integer $m$ such that $a^{m}$ is unit-regular. A ring $R$ is said to be unit $\pi$-regular if every element of $R$ is unit $\pi$-regular [6].

Theorem 3.4. The following are equivalent for a ring $R$ :

(1) $R / J$ is unit $\pi$-regular and idempotents lift modulo $J$.

(2) For any $a \in R$, there exist a positive integer $n$ and a unit-regular element $d \in R$ (resp. $\left.d \in a^{n} R\right)$ such that $a^{n}-d \in J$.

(3) For any $a \in R$, there exist a positive integer $n$ and a unit $\pi$-regular element $d \in R$ (resp. $d \in a^{n} R$ ) such that $a^{n}-d \in J$.

(4) For any $a \in R$, there exist a positive integer $n$, an idempotent $e \in R$ and $a$ unit $b \in R$ such that $e \in a^{n} R,(1-e) a^{n} \in J$ and $b a^{n}-\left(b a^{n}\right)^{2} \in J$.

(5) For any $a \in R$, there exist a positive integer $n$, an idempotent $e \in R$ and $a$ unit $b \in R$ such that $e \in R a^{n}, a^{n}(1-e) \in J$ and $a^{n} b-\left(a^{n} b\right)^{2} \in J$.

Proof. (1) $\Rightarrow(2)$ Let $a \in R$. Then there exists a positive integer $n$ such that $\bar{a}^{n}$ is unit-regular in $R / J$. Let $\bar{u}$ be a unit in $R / J$ such that $\bar{a}^{n}=\bar{a}^{n} \overline{u a}^{n}$. Since idempotents lift strongly modulo $J$, there exists an idempotent $e$ of $a^{n} R$ such that $\bar{a}^{n} \bar{u}=\bar{e}$. Hence, we have that $a^{n}-e u^{-1} \in J$, where $e u^{-1}$ is unit-regular.

$(2) \Rightarrow(3)$ It is obvious.

$(3) \Rightarrow(1)$ By a proof similar to that of $(2) \Rightarrow(1)$ in Theorem 3.1.

$(1) \Rightarrow(4)$ By a proof similar to that of $(1) \Rightarrow(4)$ in Theorem 2.1.

$(4) \Rightarrow(1)$ By [28, Proposition 4.1 and Theorem 4.4], idempotents lift modulo $J$. By a proof similar to that of $(4) \Rightarrow(1)$ in Theorem 2.1, the proof follows.

$(1) \Leftrightarrow(5)$ Follows from the symmetry of the condition (1).

We call a ring $R$ semi unit $\pi$-regular if $R$ satisfies one of the equivalent conditions of Theorem 3.4.

Corollary 3.5. If $R$ is a semi unit $\pi$-regular ring, then so is every homomorphic image of $R$ and so is every subring of the form eRe, where $e^{2}=e$.

Proof. By a proof similar to Corollary 2.4, every homomorphic image of $R$ is semi unit $\pi$-regular. For any idempotent $e \in R, e R e$ is semi unit- $\pi$-regular by [6, Theorem 1.2] and [21, Corollary 6].

An element $a$ of a ring $R$ is called strongly $\pi$-regular if there exist a positive integer $n$ and $x \in R$ such that $a^{n}=a^{n+1} x$ and $a^{n}=x a^{n+1}$. In [3, Corollary of Theorem 3], it is proved that a strongly $\pi$-regular element is $\pi$-regular. A ring $R$ is called strongly $\pi$-regular if every element of $R$ is strongly $\pi$-regular. Clearly, any strongly regular ring is strongly $\pi$-regular. Any regular element of 
a strongly $\pi$-regular ring is unit-regular, because strongly $\pi$-regular rings have stable range 1 . Hence, any strongly $\pi$-regular ring is unit $\pi$-regular. According to [19, Proposition 1], if $R$ is strongly $\pi$-regular ring, then, for any $a \in R$, there exists a positive integer $n$ such that $a^{n}=e u=u e$ for some idempotent $e \in R$ and some unit $u \in R$. Note that $R$ is strongly $\pi$-regular if and only if, for any $a \in R$, there exist a positive integer $n$ and a strongly $\pi$-regular element $d$ such that $a^{n}-d=0$. For the other characterizations we refer the reader to [23, Theorems 5.1 and 5.9].

Theorem 3.6. The following are equivalent for a ring $R$ :

(1) $R / J$ is strongly $\pi$-regular and idempotents lift modulo $J$.

(2) For any $a \in R$, there exist a positive integer $n$, an idempotent $e \in R$ (resp. $e \in a^{n} R$ ) and a unit $u \in R$ such that $a^{n}-e u \in J$ and $e u-u e \in J$.

(3) For any $a \in R$, there exist a positive integer $n$, an idempotent $e \in a^{n} R$ and $a$ unit $u \in R$ such that $(1-e) a^{n} \in J$ and $\bar{a}^{n} \bar{u}=\bar{u} \bar{a}^{n}$ is an idempotent of $R / J$.

(4) For any $a \in R$, there exist a positive integer $n$, an idempotent $e \in R a^{n}$ and $a$ unit $u \in R$ such that $a^{n}(1-e) \in J$ and $\bar{a}^{n} \bar{u}=\bar{u} \bar{a}^{n}$ is an idempotent of $R / J$.

Proof. (1) $\Rightarrow(2)$ Let $\bar{a}$ be in $R / J$. By hypothesis, there exists a positive integer $n$ such that $\bar{a}^{n}=\overline{e u}=\overline{u e}$, where $\bar{e} \in R / J$ is an idempotent and $\bar{u} \in R / J$ is a unit. Note also that $u$ is a unit in $R$. Since $\bar{e}=\bar{a}^{n} \overline{u^{-1}}$ and idempotents lift strongly modulo $J$, we can assume that $e$ is an idempotent of $a^{n} R$. Then we obtain that $a^{n}-e u \in J$ and $e u-u e \in J$.

$(2) \Rightarrow(1)$ Let $a \in R$. Choose $n, e$ and $u$ as in (2). Then $\bar{a}^{n}=\bar{a}^{n} \bar{e}=$ $\bar{a}^{n} \bar{a}^{n} \bar{u}^{-1}=\bar{a}^{n+1}\left(\bar{a}^{n-1} \bar{u}^{-1}\right)$ which means that $\bar{a}^{n}$ is a strongly $\pi$-regular element. Finally, idempotents lift modulo $J$ by Theorem 3.4.

$(1) \Rightarrow(3)$ Let $\bar{a} \in R / J$. Since $R / J$ is strongly $\pi$-regular, there exist a positive integer $n$ and a unit $\bar{u}$ in $R / J$ such that $\bar{a}^{n} \bar{u}$ is an idempotent and $\overline{a u}=\overline{u a}$. By $[21$, Lemma 5 and Corollary 6$]$, there exists an idempotent $e$ in $a^{n} R$ such that $\bar{e}=\bar{a}^{n} \bar{u}$. Then clearly, $\bar{a}^{n} \bar{u}=\bar{u} \bar{a}^{n}$ is an idempotent of $R / J$ and $(1-e) a^{n} \in J$.

$(3) \Rightarrow(1)$ Let $\bar{a} \in R / J$ and choose $e$ and $u$ as in (3). We can write $e=a^{n} r u$, where $r \in R$, because $a^{n} R=a^{n} R u$. Then $\bar{a}^{n}=\bar{e} \bar{a}^{n}$ implies that $\bar{a}^{n} \bar{u} \bar{a}^{n}=\bar{a}^{n}$. By hypothesis, $\bar{a}$ is a strongly $\pi$-regular element.

$(1) \Leftrightarrow(4)$ Follows from the symmetry of the condition (1).

We call $R$ a semi strongly $\pi$-regular ring if it satisfies one of the equivalent conditions of Theorem 3.6.

Theorem 3.7. If, for any $a \in R$, there exist a positive integer $n$ and a strongly $\pi$-regular element $d$ such that $a^{n}-d \in J$, then $R / J$ is strongly $\pi$-regular and idempotents lift modulo $\mathrm{J}$.

Proof. Let $a \in R, n$ be a positive integer and $d$ be a strongly $\pi$-regular element of $R$ such that $a^{n}-d \in J$. Then $d$ is $\pi$-regular, so there exists a positive 
integer $m$ such that $d^{m}$ is regular. This implies that $a^{n m}-d^{m} \in J$. By $[28$, Theorem 4.4], $R / J$ is $\pi$-regular and idempotents lift modulo $J$. On the other hand, $\bar{a}^{n}=\bar{d}$ is strongly $\pi$-regular and so there exist a positive integer $t$ and $\bar{x} \in R / J$ such that $\bar{a}^{n t}=\bar{a}^{n(t+1)} \bar{x}=\bar{a}^{n t+1}\left(\bar{a}^{n t+n-1} \bar{x}\right)$. Hence, $\bar{a}$ is strongly $\pi$-regular.

Corollary 3.8. If $R$ is a semi strongly $\pi$-regular ring, then so is every homomorphic image of $R$ and so is every subring of the form eRe, where $e^{2}=e$.

Proof. Any homomorphic image of a semi strongly $\pi$-regular ring is also semi strongly $\pi$-regular by Theorem 3.6. For any idempotent $e \in R, e R e$ inherits the strong $\pi$-regularity from a ring $R$. Hence, this fact together with [21, Corollary $6]$ implies that $e R e$ is semi strongly $\pi$-regular.

An element $a \in R$ is called right weakly $\pi$-regular if there exists a positive integer $n$ such that $a^{n} R=\left(a^{n} R\right)^{2}$. A ring $R$ is called right weakly $\pi$-regular if every element of $R$ is right weakly $\pi$-regular [13]. Note that $R$ is right weakly $\pi$-regular if and only if, for any $a \in R$, there exist a positive integer $n$ and a right weakly $\pi$-regular element $d \in R$ such that $a^{n}-d=0$. We consider the following generalization of weak $\pi$-regularity:

(*) For any $a \in R$ there exist a positive integer $n$ and a right weakly $\pi$-regular element $d \in R$ such that $a^{n}-d \in J$.

Any local ring satisfies $(*)$. For, let $x \in R$. If $x \in J$, then $x-0 \in J$. If $x \notin J$, then $x$ is a unit and so is weakly $\pi$-regular; hence $x-x=0 \in J$ gives the result.

There exists a ring satisfying $(*)$ but which is not right weakly $\pi$-regular. Let $D$ be a division ring and $R$ be the power series ring with an indeterminate $x$ over $D$. Since $R$ is local, it satisfies $(*)$, but $x^{n} \notin x^{n} R x^{n} R$ for all positive integer $n$.

Theorem 3.9. If $R$ satisfies (*), then $R / I$ is right weakly $\pi$-regular for any ideal $I$ of $R$.

Proof. If $\bar{a} \in R / I$, then there exist a positive integer $n$ and a right weakly $\pi-$ regular element $d \in R$ such that $\bar{a}^{n}=\bar{d}$. Also, there exist a positive integer $m$ and $b \in R d^{m} R$ such that $d^{m}=d^{m} b$. Since $\bar{a}^{n m}=\bar{d}^{m}$, we have that $\bar{b} \in \bar{R} \bar{a}^{n m} \bar{R}$ and that $\bar{d}^{m}=\bar{a}^{n m}=\bar{a}^{n m} \bar{b}$. Thus, $\bar{a}$ is a right weakly $\pi-$ regular element.

The results, we have obtained up to now, give rise to the following question:

Question. If $R$ satisfies $(*)$, then do idempotents lift modulo $J$ ?

If $R$ is right weakly $\pi$-regular, then $J$ is nil (see [23, 4.2]). But if $R$ satisfies $(*)$, then $J$ need not be nil, for example the Jacobson radical of the local ring $\mathbb{Z}_{(p)}$ is not nil.

Since $\pi$-regular rings are exchange, semi $\pi$-regular rings are exchange (see $[30$, p. 663$])$. But we are able to give an example showing that a semi right 
weakly $\pi$-regular ring $R$ (i.e., if $R / J$ is right weakly $\pi$-regular and idempotents lift modulo $J$ ) need not be exchange (Example 3.17).

In addition, we now consider another generalization of weak $\pi$-regularity. (**) For any $a \in R$, there exist a positive integer $n$ and $x \in R a^{n} R$ such that $a^{n}-a^{n} x \in N^{*}(R)$, where $N^{*}(R)$ denotes the upper nil radical of $R$.

Proposition 3.10. $R$ satisfies $(* *)$ if and only if $R / J$ is right weakly $\pi-r e g u l a r$ and $J$ is nil.

Proof. Assume that $R$ satisfies (**). Let $a \in J$. Then there exist a positive integer $n$ and $x \in R a^{n} R$ such that $b=a^{n}-a^{n} x \in N^{*}(R)$. So $b(1-x)^{-1}=$ $a^{n}(1-x)(1-x)^{-1}=a^{n} \in N^{*}(R)$, entailing that $a$ is nilpotent. Thus $J$ is nil. Clearly, $R / J$ is right weakly $\pi$-regular. The converse is obvious.

Recall that a ring $R$ is called right weakly regular if $B^{2}=B$ for every principal right ideal $B$ of $R$. A similar result holds for a right weakly regular ring:

Proposition 3.11. For any $a \in R$, there exists $x \in R a R$ such that $a-a x \in$ $N^{*}(R)$ if and only if $R / J$ is right weakly regular and $J$ is nil.

Next, we consider rings with the property that for any $a \in R$, there exist an idempotent $e \in R$ and a positive integer $n$ such that $a^{n}-e \in J$.

It is easy to see that $R / J$ is a Boolean ring and idempotents lift modulo $J$ if and only if for any $a \in R$, there exists an idempotent $e \in R$ such that $a-e \in J$.

An element $e \in R$ is said to be a near idempotent if $e^{n}$ is an idempotent for some positive integer $n$. Following [10], $R$ is called Eulerian if every element of $R$ is a near idempotent.

Proposition 3.12. The following are equivalent for a ring $R$ :

(1) $R / J$ is Eulerian and idempotents lift modulo $J$.

(2) For any $a \in R$, there exist an idempotent $e \in R$ (resp. $e \in a^{n} R, e \in$ $\left.a^{n} R a^{n}\right)$ and a positive integer $n$ such that $a^{n}-e \in J$.

(3) $R$ is semi strongly $\pi$-regular and the set of units of $R / J, U(R / J)$, is a torsion group.

Proof. (1) $\Leftrightarrow$ (3) follows from the fact that $R$ is Eulerian if and only if $R$ is strongly $\pi$-regular and $U(R)$ is a torsion group ([10, Proposition 2.3]). (1) $\Rightarrow(2)$ follows from definitions and [21, Lemma 5]. For $(2) \Rightarrow(1), R / J$ is clearly Eulerian and since $R$ is semi $\pi$-regular, idempotents lift modulo $J$ by [28, Theorem 4.4].

Clearly, semi strongly regular $\Rightarrow$ semi strongly $\pi$-regular $\Rightarrow$ semi unit $\pi-$ regular $\Rightarrow$ semi $\pi$-regular $\Rightarrow$ semi right weakly $\pi$-regular. The following examples show that the reverse of the implications are not true in general, but we couldn't provide an example of a semi unit $\pi$-regular ring that is not semi strongly $\pi$-regular. Recall that a ring is called 2-primal if its prime radical (i.e., lower nilradical) coincides with the set of all nilpotent elements. 
Examples 3.13. (1) Let $S$ be a 2-primal strongly $\pi$-regular ring (e.g., $U_{2}(F)$ with $F$ a field) and $R=M_{n}(S)$ where $n$ is a positive integer. Then $R$ is strongly $\pi$-regular by [14, Theorem 1] and so $R / J$ is strongly $\pi$-regular but $R / J$ need not be strongly regular because if $a=\left(\begin{array}{ll}0 & 1 \\ 0 & 0\end{array}\right)$, then $a \notin J$ and $(a+J)^{2}=0$. Since $J$ is nil ( $R$ is right weakly $\pi$-regular), $R$ is semi strongly $\pi$-regular but not semi strongly regular.

(2) [12] Let $F$ be a field and $T=F[[t]]$ be the ring of formal power series over $F$ in an indeterminate $t$, and let $K$ denote the quotient field of $T$. Let $S=\left\{x \in \operatorname{End}_{F}(T) \mid(x-a)\left(t^{n} T\right)=0\right.$ for some $a \in K$ and $\left.n>0\right\}$. By [12, Example 4.26], for each $x \in S$ there is a unique element $\varphi x \in K$ such that $(x-\varphi x)\left(t^{n} T\right)=0$ for some $n>0$. Since $K$ is commutative, the map $\varphi: S \rightarrow K$ also defines a ring map $\varphi: S^{o p} \rightarrow K$, where $S^{o p}$ denotes the opposite ring of $S$. Consequently, the set $R=\left\{(x, y) \in S \times S^{o p} \mid \varphi x=\varphi y\right\}$ is a subring of $S \times S^{o p}$. Then $R$ is regular, and by [16, Example 2.27] $R$ is not unit $\pi$-regular. Hence $R$ is semi $\pi$-regular but not semi unit $\pi$-regular.

(3) The ring $R$ in Example 3.17, to follow, is semi right weakly $\pi$-regular but not semi $\pi$-regular because it is not an exchange ring.

In [10, Theorem 3.1], it is proved that if $R$ is an abelian ring, then $R$ is strongly $\pi$-regular if and only if $R / N(R)$ is regular and $N(R)=J$, where $N(R)$ stands for the set of all nilpotent elements of $R$. Then we have the following result.

Theorem 3.14. If $R$ is an abelian ring, then the following are equivalent:

(1) $R$ is semi strongly $\pi$-regular.

(2) $R$ is semiregular.

(3) $R$ is semi strongly regular.

(4) $R$ is semi unit-regular.

(5) $R$ is semi one-sided unit-regular.

(6) $R$ is semi unit $\pi$-regular.

(7) $R$ is semi $\pi$-regular.

Proof. Firstly note that $R / J$ is abelian since idempotents lift modulo $J$. By [10, Theorem 3.1], $R$ is semi strongly $\pi$-regular if and only if $R / J$ is regular, $N(R / J)=0$ and idempotents lift modulo $J$, if and only if $R$ is semi strongly regular (since strongly regular rings coincide with regular reduced rings), if and only if $R$ is semiregular. Hence, (1)-(3) are equivalent. The implication $(7) \Rightarrow(1)$ is obtained from the fact that $\pi$-regular abelian rings are strongly $\pi$-regular. The other statements are clearly equivalent.

A ring $R$ is called right quasi-duo if every maximal right ideal is a two-sided ideal.

Proposition 3.15. If $R$ is a right quasi-duo ring, then the following are equivalent:

(1) $R$ is semi strongly $\pi$-regular. 
(2) $R$ is semi $\pi$-regular.

(3) $R$ is semi right weakly $\pi$-regular.

(4) $R$ is semi strongly regular.

(5) $R$ is semi unit-regular.

(6) $R$ is semi one-sided unit-regular.

(7) $R$ is semiregular.

(8) $R$ is semi unit $\pi$-regular.

Proof. (4) $\Rightarrow(5) \Rightarrow(6) \Rightarrow(7) \Rightarrow(2)$ and (1) $\Rightarrow$ (8) are obvious.

$(1) \Leftrightarrow(2) \Leftrightarrow(3)$ By $[29], R / J$ is a right quasi-duo ring. Then $R / J$ is right weakly $\pi$-regular if and only if $R / J$ is strongly $\pi$-regular ([15, Theorem 7$]$ ), if and only if $R / J$ is $\pi$-regular.

(2) $\Rightarrow$ (4) Since semi $\pi$-regular rings are exchange, we have that $R / J$ is strongly $\pi$-regular if and only if $R / J$ is strongly regular by [30, Theorem 3.8].

$(8) \Rightarrow(2)$ Follows from Theorems 3.1 and 3.4.

Example 3.16. There exists a semi right weakly $\pi$-regular ring that is not semi $\pi$-regular and not right quasi-duo.

Proof. [15, Example 4] Let $D$ be a simple domain that is not a division ring. Consider the ring

$$
R=\left\{\left(\begin{array}{ll}
a & b \\
0 & a
\end{array}\right) \mid a, b \in D\right\} .
$$

$R$ is right weakly $\pi$-regular and so $R / J$ is right weakly $\pi$-regular. Since $J=\left(\begin{array}{ll}0 & D \\ 0 & 0\end{array}\right)$ is nil, idempotents lift modulo $J$. Hence, $R$ is semi right weakly $\pi$-regular. But $R / J$ is not $\pi$-regular. For, let $\left(\begin{array}{ll}a & 0 \\ 0 & a\end{array}\right)+J$, where $a$ is a non-zero non-unit in $D$. If it was a $\pi$-regular element of $R / J$, then $a$ would be a unit of $D$, which is a contradiction.

Moreover, $R$ is not a right quasi-duo ring because of [15, Theorem 7].

Example 3.17. There exists a semi right weakly $\pi$-regular ring that is not exchange.

Proof. The ring $R$ in Example 3.16 is semi right weakly $\pi$-regular. We claim that $R$ is not exchange. It is known that a ring $R$ is exchange if and only if $\bar{R}=R / J$ is exchange and idempotents lift modulo $J$. Therefore it is enough to show that $\bar{R}$ is not exchange. Let $a$ be a non-zero element in $D$ such that $a$ and $1-a$ are both not right unit (for the existence of such an element consider the element $x$ in the first Weyl algebra over a field of zero characteristic). Suppose that $\bar{R}$ is exchange. Then there exists an idempotent $\bar{e} \in \bar{k} R$ such that $(\overline{1}-\bar{e}) \in(\overline{1}-\bar{k}) \bar{R}$, where $\bar{k}=\left(\begin{array}{cc}a & 0 \\ 0 & a\end{array}\right)+J$. Note that since $\bar{R} \cong D$, the only idempotents in $\bar{R}$ is $\overline{0}$ and $\overline{1}$. If $\bar{e}=\overline{0}$, then $\overline{1} \in(\overline{1}-\bar{k}) \bar{R}$ so $(\overline{1}-\bar{k}) \bar{R}=\bar{R}$. It follows that there exists an element $\bar{y} \in \bar{R}$ such that $(\overline{1}-\bar{k}) \bar{y}=\overline{1}$. This gives that there exists an element $b \in D$ such that $(1-a) b=1$, which is a contradiction. If $\bar{e}=\overline{1}$, then we obtain that $a$ is a right unit in a similar way. Thus, $R$ is not an exchange ring. 
A ring $R$ is called a $p m$-ring if every prime ideal of $R$ is maximal. The relationship between $\mathrm{pm}$-rings and various generalizations of regular rings has been given by many authors (see, for example, $[15,29]$ ). We wonder if any semi (*)-regular rings is a $p m$-ring or not. The answer is negative:

Example 3.18. A semi strongly regular ring need not be a pm-ring.

Proof. Denote by $U_{n}$ the $2^{n}$ by $2^{n}$ upper triangular matrix over a ring $S$, where $n$ is a positive integer. We construct a prime $\pi$-regular ring with the help of [17, Example 1.2 and Proposition 1.3].

Let $S$ be a division ring. Define a map $\sigma: U_{n} \rightarrow U_{n+1}$ by $A \rightarrow\left(\begin{array}{cc}A & 0 \\ 0 & A\end{array}\right)$, then $U_{n}$ can be considered as a subring of $U_{n+1}$ via $\sigma$ (i.e., $A=\sigma(A)$ for $A \in U_{n}$ ). Set $R$ be the direct limit of the direct system $\left(U_{n}, \sigma_{i j}\right)$ with $\sigma_{i j}=\sigma^{j-i}$. Then $R$ is a prime ring by [17, Proposition 1.3]. Since every $U_{n}$ is $\pi$-regular, $R$ is also $\pi$-regular by the definition of $R$.

Consider the subset $I=\{A \in R \mid$ the diagonal entries of $A$ are all zero $\}$ of $R$. Then $I$ is a nil ideal of $R$ such that $R / I$ is isomorphic to a direct product of division rings. This implies that $R / I$ is strongly regular and the Jacobson radical of $R$ is $I$. Consequently $R$ is semi strongly regular with $J$ is nil.

But the zero ideal of $R$ is prime and not maximal since $I$ is a proper ideal of $R$.

Remark. Let $R$ be a semi right weakly $\pi$-regular ring with $J$ nil. If $R$ is $2-$ primal, then $R$ is a $p m-$ ring by [15, Proposition 5]. Note that $R$ in Example 3.18 is not 2-primal by [17, Example 1.2].

Acknowledgements. The authors thank the referee for his/her very careful reading of the manuscript and suggestions that improved the paper. The second named author was supported by Basic Science Research Program through the National Research Foundation of Korea(NRF) funded by the Ministry of Education, Science and Technology(No. 20110004745).

\section{References}

[1] P. Ara, Strongly $\pi$-regular rings have stable range one, Proc. Amer. Math. Soc. 124 (1996), no. 11, 3293-3298.

[2] R. F. Arens and I. Kaplansky, Topological representations of algebras, Trans. Amer. Math. Soc. 63 (1948), 457-481

[3] G. Azumaya, Strongly $\pi$-regular rings, J. Fac. Sci. Hokkaido Univ. Ser. I 13 (1954), 34-39.

[4] V. P. Camillo and H. P. Yu, Stable range one for rings with many idempotents, Trans. Amer. Math. Soc. 347 (1995), no. 8, 3141-3147.

[5] _ Exchange rings, units and idempotents, Comm. Algebra 22 (1994), no. 12, 4737-4749.

[6] H. Chen, A note on the $\pi$-regularity of rings, Chinese Quart. J. Math. 13 (1998), no. $2,67-71$.

[7] _ Exchange rings satisfying unit 1-stable range, Kyushu J. Math. 54 (2000), 1-6.

[8] _ On strongly stable rings, Comm. Algebra 31 (2003), no. 6, 2771-2789.

[9] H. Chen and M. Chen, On semiregular rings, New Zealand J. Math. 32 (2003), 11-20. 
[10] A. Y. M. Chin, A note on strongly $\pi$-regular rings, Acta Math. Hungar. 102 (2004), no. 4, 337-342.

[11] P. Crawley and B. Jonsson, Refinements for infinite direct decompositions of algebraic systems, Pacific J. Math. 14 (1964), 797-855.

[12] K. R. Goodearl, Von-Neumann Regular Rings, Pitman Publishing Limited, London, 1979.

[13] V. Gupta, Weakly $\pi$-regular rings and group rings, Math. J. Okayama Univ. 19 (1977), no. 2, 123-127.

[14] Y. Hirano, Some studies on strongly $\pi$-regular rings, Math. J. Okayama Univ. 20 (1978), no. 2, 141-149.

[15] C. H. Hong, N. K. Kim, T. K. Kwak, and Y. Lee, On weak $\pi$-regularity of rings whose prime ideals are maximal, J. Pure Appl. Algebra 146 (2000), no. 1, 35-44.

[16] Q. Huang and J. Chen, $\pi-$ morphic rings, Kyungpook Math. J. 47 (2007), no. 3, 363-372.

[17] S. U. Hwang, Y. C. Jeon, and Y. Lee, Structure and topological conditions of NI-rings, J. Algebra 302 (2006), no. 1, 186-199.

[18] Q. Li and W. T. Tong, Weak cancellation of modules and weakly stable range conditions for exchange rings, Acta Math. Sinica 45 (2002), no. 6, 1121-1126.

[19] W. K. Nicholson, Strongly clean rings and fitting's lemma, Comm. Algebra, 27 (1999), no. 8, 3583-3592.

[20] W. K. Nicholson and M. F. Yousif, Quasi-Frobenius Rings, Cambridge Tracts in Math. 158., Cambridge University Press, Cambridge, UK, 2003.

[21] W. K. Nicholson and Y. Zhou, Strong lifting, J. Algebra 285 (2005), no. 2, 795-818.

[22] V. S. Ramamurthi, Weakly regular rings, Canad. Math. Bull. 16 (1973), 317-321.

[23] A. A. Tuganbaev, Semiregular, weakly regular and $\pi$-regular rings, J. Math. Sci. (New York) 109 (2002), no. 3, 1509-1588.

[24] L. N. Vaserstein, Bass's first stable range condition, J. Pure Appl. Algebra 34 (1984), no. 2-3, 319-330.

[25] R. B. Warfield, Exchange rings and decomposition of modules, Math. Ann. 199 (1972), $31-36$.

[26] T. S. Wu, Weak cancellation of modules and the weak stable range one condition, Nanjing Daxue Xuebao Shuxue Bannian Kan 11 (1994), no. 2, 109-116.

[27] _ Inner weak cancellation of modules, Nanjing Daxue Xuebao Shuxue Bannian Kan 13 (1996), no. 1, 54-57.

[28] G. Xiao and W. Tong, Generalizations of semiregular rings, Comm. Algebra 33 (2005), no. 10, 3447-3465.

[29] H. P. Yu, On quasi-duo rings, Glasg. Math. J. 37 (1995), no. 1, 21-31.

[30] _ On the structure of exchange rings, Comm. Algebra 25 (1997), no. 2, 661-670.

PINAR AYDOĞDU

Department of Mathematics

HACETTEPE UNIVERSITY

06800 Beytepe Ankara, Turkey

E-mail address: paydogdu@hacettepe.edu.tr

YANG LEE

Department of Mathematics Education

Pusan National University

Pusan 609-735, Korea

E-mail address: ylee@pusan.ac.kr 


\author{
A. ÇIĞDEM ÖzCAN \\ Department of Mathematics \\ HACETTEPE UNIVERSITY \\ 06800 Beytepe Ankara, Turkey \\ E-mail address: ozcan@hacettepe.edu.tr
}

